\title{
Spatial distribution of charge-density-wave excitations due to current conversion near contacts
}

\author{
F.Ya. NAD', M.E. ITKIS, P. MONCEAU* and M. RENARD* \\ Institute of Radioengineering \& Electronics, Russian Academy of Sciences, 103907 Moscow, Russia \\ * CRTBT, Laboratoire associé à l'UJF, BP. 166, 38042 Grenoble cedex 9, France
}

\begin{abstract}
The non uniform spatial distribution of the electric field $\mathrm{E}$ along the quasi-onedimensional conductor $\left(\mathrm{o}-\mathrm{TaS}_{3}\right)$ has been studied in the low-temperature range $(\mathrm{T}<50 \mathrm{~K})$ for a d.c. voltage $\mathrm{V}$ applied between current terminals less than the threshold value $V_{T}$. We have found a strong increase of $\mathrm{E}$ near negative terminal with respect to its value in the middle of the sample, on the contrary $E$ is reduced near the positive terminal. Such distribution of $\mathrm{E}$ is supposed to be related to the formation of two Schottky barriers at the metalPeierls conductor contacts. With increasing V, both the size of non-uniform $\mathrm{E}$ region and appropriate band bending are enlarged. We suggest that these effects are related to space rearrangement of CDW deformation (solitons and dislocations) which also exist in CDW at $\mathrm{V}<\mathrm{V}_{\mathrm{T}}$. In the range $\mathrm{V}>\mathrm{V}_{\mathrm{T}}$ the electric field increases near both contacts and spatial distribution of $\mathrm{E}$ becomes more symmetrical.
\end{abstract}

\section{1 - INTRODUCTION}

It is now well known that under the influence of various forces such as electric field, thermal gradients ..., the charge density waves (CDW) in quasi one-dimensional conductors develop elastic and platic deformations [1,2]. Mihaly and Janossy [3] have measured the remanent CDW deformation after the application of current pulses larger than the threshold current $\mathrm{I}_{\mathrm{T}}$ and they have shown that the CDW is stretched near one terminal and compressed near another terminal. The spatial distribution of the remanent CDW deformation has been measured by means of laser beam scanning [4] and with a mobile contact [5]. The same technique of mobile contact has been used for measuring the spatial distribution of the potential $\mathrm{V}(\mathrm{x})$ along an orthorhombic $\mathrm{TaS}_{3}$ sample with non zero current [6]. While at $T_{c}=120 \mathrm{~K}$ an additional voltage $\delta \mathrm{V}$ with the same magnitude is shown to appear near both terminals, at $\mathrm{T}=75 \mathrm{~K}$ the distribution of $\delta \mathrm{V}(\mathrm{x})$ becomes asymmetrical and $\delta \mathrm{V}$ exceeds the $\Delta$ /e value where $\Delta$ is the energy gap.

In the present work, we report measurements of the spatial distribution of the electric field $\mathrm{E}$, between two current terminals in the sliding mode of orthorhombic $\mathrm{TaS}_{3}$ samples (typical size : $10 \times 1 \times 1000 \mu \mathrm{m}^{3}$ ) using a multicontact configuration. We have performed these measurements in an extended temperature range : $30 \mathrm{~K}<\mathrm{T}<120 \mathrm{~K}$. We show that the size of the region of non uniform electric field near terminals depend on magnitude and direction of the applied current.

\section{2 - EXPERIMENTAL RESULTS}

By means of mobile contact the spatial distribution of measured potentials, between nearby electrodes (distances of $40 \mu \mathrm{m}$ ) is converted in a local field distribution using $\mathrm{E}(\mathrm{x})=$ $\delta \mathrm{V} / \delta \mathrm{x}$. This field which is expected to be constant in an uniform conductor for a given current shows in orthorhombic $\mathrm{TaS}_{3}$ a plateau $\mathrm{E}_{\mathrm{O}}$ in a large central part of the sample and variations in the vicinity of the injection electrodes, over a characteristic length $\mathrm{L}$. The electrical conductivity $\sigma(\mathrm{E})$ exhibits the typical behaviour $[7,8]$ namely it is ohmic for $\mathrm{E}_{<}<\mathrm{E}_{\mathrm{T}}^{\prime}$ and it shows a weak non-linearity above the first threshold field $E_{T}^{\prime}$ while strong non linearity only occurs above $\mathrm{E}_{\mathrm{T}}<\mathrm{E}_{\mathrm{T}}^{\prime}$ (Fig. $1 \mathrm{~b}$ ).

The main results can be summarized as follows : 
- at high temperature (namely $\mathrm{T} \gtrsim 100 \mathrm{~K}$ ) $\mathrm{E}(\mathrm{x})$ is symmetrical and shows an increase near both terminals $\left(\Delta \mathrm{E} / \mathrm{E}_{\mathrm{o}} \sim 5 \%\right)$,

- at lower temperature for $\mathrm{E}_{\mathrm{T}}<\mathrm{E}<\mathrm{E}_{\mathrm{T}}$ the distribution of $\mathrm{E}(\mathrm{x})$ becomes aysmmetrical with a large growth of electric field when approaching the negative contact and a decrease of electric field but with a smaller amplitude near the positive one (Fig. 1a).

The asymmetry is described by the ratio $\Delta \mathrm{E} / \mathrm{E}_{\mathrm{O}}$ where $\Delta \mathrm{E}$ is equal to the difference between the field $\mathrm{E}_{\mathrm{m}}$ measured at the shortest distance near the current contact and $\mathrm{E}_{\mathrm{O}}$. Fig. $2 \mathrm{~b}$ shows that $\Delta \mathrm{E} / \mathrm{E}_{\mathrm{O}}$ have different signs at oppositive polarity contacts. The amplitude of $\Delta \mathrm{E} / \mathrm{E}_{\mathrm{o}}$ grow with increasing $\mathrm{E}$ and they reach extrema at $\mathrm{E}=\mathrm{E}_{\mathrm{T}}$. For $\mathrm{E}>\mathrm{E}_{\mathrm{T}}$ the $\mathrm{E}(\mathrm{x})$ distribution becomes more and more symmetrical.

One can derive the length $L$ on which the non-uniformity of the electric field spreads out from the contacts within the sample. We determine $\mathrm{L}$ as the distance between the current contact and a point in the sample where magnitude $\Delta \mathrm{E}$ increases by $10 \%$ with respect to the electric field $E_{o}$ in the middle of the sample. The $L(E)$ dependences for the negative contact are shown in figures 1 (b) et $2(a)$ for samples 1 and 2 . $L$ is maximum at $E \simeq E_{T}$ and reaches a value of $\sim 500 \mu \mathrm{m}$ for sample 1 and $\sim 300 \mu \mathrm{m}$ for sample 2 . We can compare the $\mathrm{L}(\mathrm{T})$ values of different samples obtained at the field $E(T)$ that correspond to an equal relative development of the non-linearity in the $\sigma(\mathrm{E}, \mathrm{T})$ dependences. Figure 3 shows the dependence of $L$ on the reciprocal of temperature $L(T)$ obtained for such $E$ magnitudes when the conductivity $\sigma$ is ten times higher than the ohmic conductivity. As can be seen, when $\mathrm{T}$ is decreased, the $\mathrm{L}$ value grows approximately by one order of magnitude and at $\mathrm{T}<50-60 \mathrm{~K}$. $\mathrm{L}$ tends to saturation at the level of several hundred of microns.
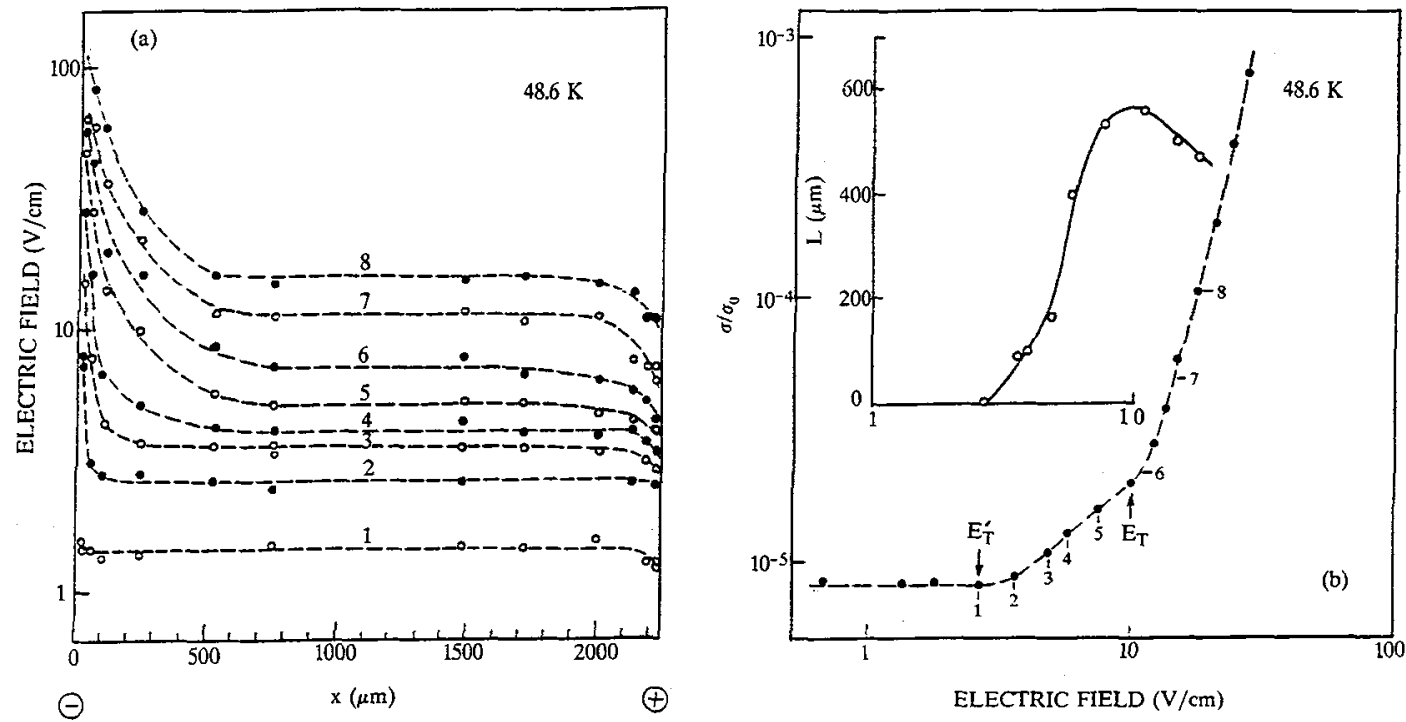

Fig. 1 - (a) Spatial distribution of electric field $\mathrm{E}(\mathrm{x})$ of orthorhombic TaS3 (sample 1 ) at $\mathrm{T}=48.6 \mathrm{~K}$ at fixed currents. (b) Conductivity $\sigma$ renormalized to its room-temperature value $\sigma_{\mathrm{o}}$ as a function of mean electric field $\mathrm{E}$ for sample 1 . The figures near the $\sigma(E)$ curve correspond to the same currents as in (a). The inset shows the length corresponding to the region of non-uniformity of $\mathrm{E}(\mathrm{x})$ near the negative current contact as a function of the mean electric field. 


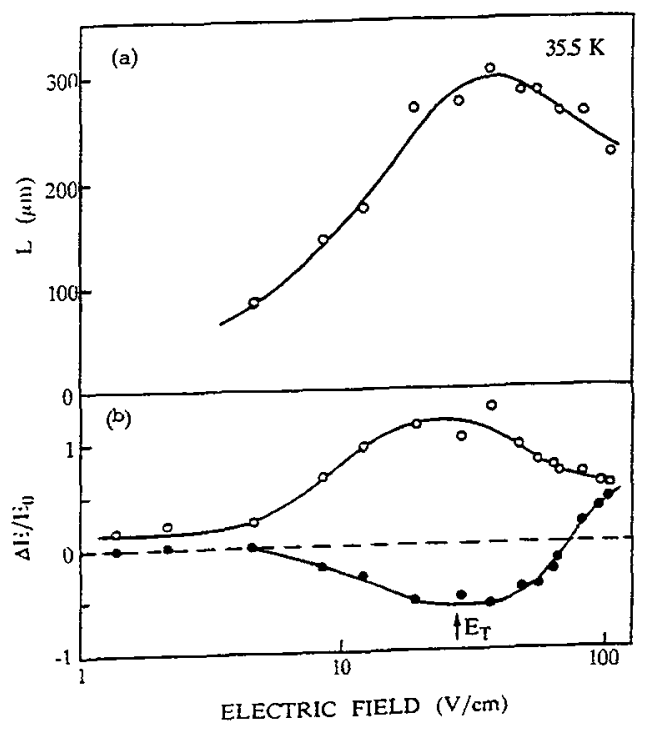

Fig. 2 a) Length $L$ corresponding to the region of nonuniformity of $E(x)$ near the negative contact, as a function of the mean electric field for 0 -TaS3 (sample 2) at $T=35.5 \mathrm{~K}$. b) Relative magnitude of the nonuniformity $\Delta \mathrm{E} / \mathrm{E}_{\mathrm{o}}$ as a function of the mean electric field for negative contact (open circles) and positive contact (filled circles). $E_{o}$ is the electric field in the middle of the sample.

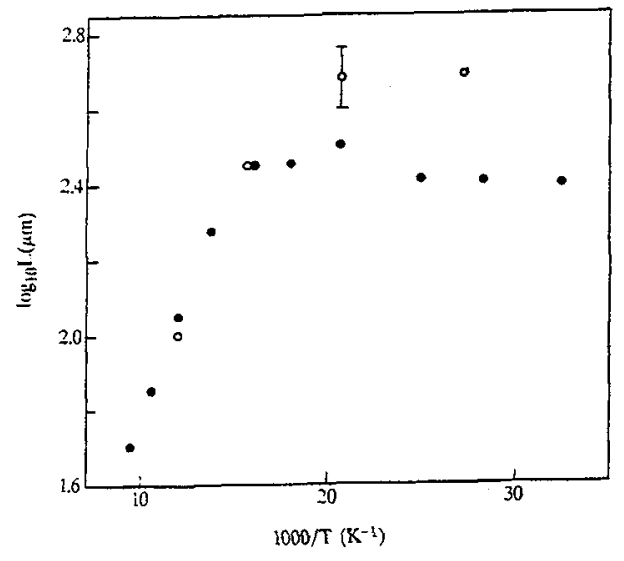

Fig. 3 - Variation of the length revealing the nonuniformity of $\mathrm{E}(\mathrm{x})$ near the negative current contact as a function of reciprocal temperature for $0-\mathrm{TaS}_{3}$, sample 1 : open circle, sample 2 : filled circles. For each temperature the field $E(x)$ is such that the conductivity $\sigma(E)$ is ten times higher than the ohmic conductivity.

\section{DISCUSSION AND CONCLUSIONS}

We will now present a qualitative explanation of the results obtained (for a complete discussion see ref. [9]). We will make the assumption that at $\mathrm{T}<\mathrm{T}_{\mathrm{p}}$ and for weak electric field, $\mathrm{E} \ll \mathrm{E}_{\mathrm{T}}$, quasi one-dimensional conductors are similar to semiconductors and that they show their specific properties due to CDW motion mainly in the high-electric-field range, E $>E_{T}$. This dual nature of these materials can be demonstrated with measurements performed at low temperatures when the free electron concentration is small. In this temperature range the contribution of collective excitations due to local CDW deformations near defects and impurities to transport phenomena exceeds the contribution of holes and electrons. These CDW excitations are amplitude and phase solitons which have a charge, effective mass and which are able of moving through the crystal as quasi particles. In particular the activation energy of phase solitons is $\simeq T_{p}$ smaller than the Peierls gap.

When a Peierls semiconductor such as $\mathrm{TaS}_{3}$ is put in a intimate contact with a metal the common chemical potential for quasi-particles must be establisehd. This can be achieved by charge transfer from the semiconductor into the metal conduction band. However, the "majority carriers" in the Peierls semiconductors, solitons and dislocations, are not able to cross the metal-semiconductor interface. Howewer, near this interface and across the crystal these solitons can dissociate into free electrons, which are able to cross the metalsemiconductor interface. The negative charge transferred to the metal electrode must be screened by the positive space charge developed within the semiconductor. As a result the depletion layer and the appropriate band bending develop within the semiconductor with a potential barrier at the metal-semiconductor interface like a Schottky barrier [10]. In the case of usual semiconductors the space charge is generated by ionized impurities. In our opinion, for a Peierls conductor the space-charge and screening effects are determined by appropriate redistribution of solitons and dislocations taking into account their pinning and depinning at the impurity sites.

We associate the asymmetrical $E(x)$ distribution with the fact that the two current contacts are biased in the opposite way. The contact near the negative electrode is biased in 
the reverse direction [10]. Under this condition the band bending, the potential non-uniformity and the $\mathrm{L}$ value are larger near the negative electrode than near the positive one. The additionnal voltage near the negative electrode $\Delta V$ can reach $600 \mathrm{mV}$ which is considerably larger than the maximum possible shift of the chemical potential level within the CDW energy gap, $\Delta / \mathrm{e} \simeq 60 \mathrm{meV}$, developed due to the CDW deformation.

As was mentioned above, at low temperatures a large part of the conductivity is associated with the motion of solitons and dislocations. With increasing $\mathrm{E}$ the electron injection across the Schottky barrier grows. According to the theory [11] every two injected electrons turn into a $2 \pi$ soliton near the contact. The solitons are added to the intrinsic solitons and dislocations which have been created under the influence of impurities and defects in the CDW superlattice. In our case the condition of injection at the negative contact which is biased in reverse direction and at the positive contact which is biased in forward direction are different. For example, electrons near the negative electrode undergo a higher electric field than near the positive one. As a result the processes of conversion of electrons to solitons can be different near these two contacts. These additional circumstances can make a contribution to the asymmetry of the $E(x)$ contribution.

At higher temperatures $(T>100 \mathrm{~K})$ the development of non-uniformity of $\mathrm{E}$ begins at $\mathrm{V}>\mathrm{V}_{\mathrm{T}}$. The distribution of $\mathrm{E}$ is symmetrical and it corresponds to increasing of $\mathrm{E}$ near both contacts. We suggest that at $\mathrm{V}>\mathrm{V}_{\mathrm{T}}$ (especially at high temperatures) the spatial distribution of $\mathrm{E}$ is governed by the voltage developed to maintain the conversion of the current of electrons in the sliding CDW condensate. In this case the phase slippage in CDW and following motion of produced solitons and dislocations along the sample are fundamental processes.

In conclusion, we have demonstrated that in $\mathrm{TaS}_{3}$ at low temperatures the electric field distribution is non-uniform and asymmetrical. The form of this distribution changes as a function of temperature and current. The data are interpreted on the basis of the suggestion that at low temperatures the dynamic properties of $\mathrm{TaS}_{3}$ are determined by the motion of solitons and dislocations. The contact phenomena at the interface between the metal electrode and the Peierls semiconductor $\mathrm{TaS}_{3}$ are shown to be the main reason for the asymmetry in the electric field distribution.

Acknowledgments - We would like to acknowledge many useful discussions with S.A. Brazowskii and S.I. Matveenko. We thank F. Levy for providing us with $\mathrm{TaS}_{3}$ samples. One of the authors (MI) is very grateful to Centre de Recherches sur Les Très Basses Températures, laboratoire associé à l'Université Joseph Fourier de Grenoble, for kind hospitality and to M.E. Gershenson for help in preparing the platinium strips on the substrate.

\section{References}

[1] Feinberg D. and Friedel J., Low-dimensional Electronic Properties of Molybdenum Bronzes and oxides . Ed. C. Schlenker (Dordrecht, Kluwer) (1989) 407.

[2] Nad' F., Charge Density Waves in Solid (Modern Problems in Condensed Matter Science 25) Ed. L.P. Gor'kov and G. Gruner (Amsterdam, Elsevier) (1989) 191.

[3] Mihaly L. and Janossy A., Phys. Rev. B30 (1984) 3530

[4] Itkis M.E., Nad' F.Ya and Pokrovskii V.Ya, Soviet Phys.-JETP 63 (1986) 177.

[5] Brown S.E., Mihaly L. and Gruner G., Solid State Commun. 58 (1986) 177.

[6] Zaitsev-Zotov S.V., Synth. Met. 29 (1989) F433.

[7] Itkis M.E., Nad'F. Ýa and Monceau P., J. Phys. ; Condensed Matter 2 ( 1990) 8327.

[8] Nad F. Ya and P. Monceau, Phys. Rev. B 46 (1992) 7413

[9] Itkis M.E., Nad' F.Ya, P. Monceau and M. Renard, J. Phys.:Condensed Matter, 5 (1993) 4631.

[10] Sze C.M., Physics of Semiconductor Devices (1969) (New York, Wiley).

[11] Brazovskii S. and Matveenko S.I., J. Physique 1 (1991) 269 and 1173. 